\title{
Optimal homotopy asymptotic method to large post-buckling deformation of MEMS
}

\author{
Nicolae Herisanu ${ }^{1,2, *}$, and Vasile Marinca ${ }^{1,2}$ \\ ${ }^{1}$ University Politehnica Timisoara, 300222 Bd. Mihai Viteazu 1, Timisoara, Romania \\ ${ }^{2}$ Center for Advanced Technical Research, Romanian Academy Branch of Timisoara, Bd. Mihai Viteazu 24, Timisoara, Romania
}

\begin{abstract}
In the present paper, the post-buckling response of an axially stressed clampedclamped actuator, modeled as a beam and subjected to a symmetric electrostatic field is analyzed. An analytical approximate method, namely the Optimal Homotopy Asymptotic Method (OHAM) is applied to the governing nonlinear integro-differential equation. The analytical results obtained through the proposed procedure show excellent agreement with numerical solution, proving the validity of the proposed procedure, which is simple and easy to use.
\end{abstract}

\section{Introduction}

Micro electromechanical systems (MEMS) have high popularity in industry and engineering, being an extension of microelectronics and integrated circuits technology. MEMS are intensively used in various fields such as wireless communication, automotive, aviation, navigation, biomedicine, consumer electronics, and so on [1]. There exists a large variety of MEMS, which may be classified as pneumatic, thermal, electrical or piezoelectric.

Several experimental or numerical studies have been conducted on dynamic behavior of MEMS devices. The mathematical study of the MEMS started by the pioneering work of Nathanson et al [2]. They considered a model of a parallel-plates capacitor of which one plate is fixed and their other is a movable mass attached to a spring. MEMS resonators have been used as transducers in mechanical microsensors [3] or as a flexible alternative to conventional large-size resonators [4]. An analytical approach and a reduced-order model to investigate the behavior of electrically actuated microbeam-based MEMS are presented in [5] by Younis et al. Nayfeh et al. [6] studied the pull-in instability in MEMS resonators and established that characteristics of the pull-in phenomenon in the presence of alternate current (AC) loads differ from those under purely direct current (DC) loads. A new test structure and method for measuring residual stresses was presented by Abu-Salih and Elata [7]. They showed that a single test structure of the proposed design may be used to measure compressive and tensile residual stress in a continuous wide range. Hu applied three analytical models, namely the full-order, the fourth-order and the third-order models and the corresponding closed form solutions for the pull-in voltages of micro beams subjected to electrostatic loads [8]. The electromechanical post- buckling response of an axial stressed clamped-clamped beam actuator subjected to a symmetric electrostatic field is analyzed by $\mathrm{Yu}$ et al. [9]. Rezazadeh et al [10] presented the static and dynamic responses of a fixedfixed and cantilever microbeam, using the lumped and the distributed models to a DC and a step DC voltage. Squeeze-film characteristics of electrostatically actuated microbeams under large DC load coupled with small AC component are presented for the first three flexural modes of vibration of the resonator operating in different ambient pressure conditions, by Chaterjee and Pokit [11]. Zhu and Espinoza presented in [12] a methodology for a coupled-field finite element analysis (FEM) to examine the performance of radio frequency MEMS switches as a function of temperature. Also, the structure of out-of-plane profile was investigated in detail. Beni and Heidari investigated the pull-in instability of microbeams with a curved grown electrode under action of electric force within the framework of von-Karman nonlinearity and the Euler-Bernoulli beam theory [15]. The effects of geometric parameters such as beam lengths, width, thickness, gaps, and size effect are discussed in detail through a numerical study. Mojahedi et al. [14] studied static pull-in instability of electrostatically-actuated microbridges and microcantilevers considering different nonlinear effects. The nonlinear differential equations are converted by means of Galerkin's decomposition method into nonlinear integro-differential equations. In [15], Singh et al. studied the linear and nonlinear frequency characteristics of non-uniform beam with tip mass using the method of multiple scales to obtain the approximate solution. The amplitude-voltage response of MEMS resonator cantilevers under soft electrostatic actuation is studied by Caruntu et al [16]. The electrostatic actuation is of the voltage of frequency near half natural frequency of the system. It is showed that the instabilities for high

\footnotetext{
* Corresponding author: nicolae.herisanu@upt.ro
} 
amplitudes of the resonator are not captured by the method of multiple scales. Daneshpajooh and Moghimi Zand used the homotopy analysis method to investigate the nonlinear oscillatory behavior of clamped-clamped initially curved micro/nano beam, under electrostatic force actuation. The influence of initial elevation, input voltage, midplane stretching are also examined. The effect of squeeze air-film and $\mathrm{AC}$ actuation voltage on dynamic stability of microswitch actuated electrostatically has been investigated in squeeze film domain by Harish et al. [19]. Using trajectories in phase plane and time history, characteristics of the pull-in phenomena have been studied in the presence of DC voltage combined with AC component.

Taking into account these considerations, the main objective of the present paper is to propose an accurate procedure to investigate the electromechanical postbuckling response of an axially stressed clampedclamped actuation, subject to a symmetric electrostatic field. The analytical approximate solutions are obtained through a version of OHAM applied to a nonlinear integral-differential equation established in [7] and [9]. Our procedure, which is independent of the presence of small or large parameters, is based on the construction and determination of the linear operator and of the auxiliary functions, combined with a convenient way to optimally control the convergence of the solution. The efficiency of the present procedure is proved, while an accurate solution is explicitly analytically obtained after only one iteration.

\section{Equation of motion}

In this section, we consider the post-buckling response of an axially stressed clamped-clamped actuator, modeled as a beam and subjected to a symmetric electrostatic field (fig.1).

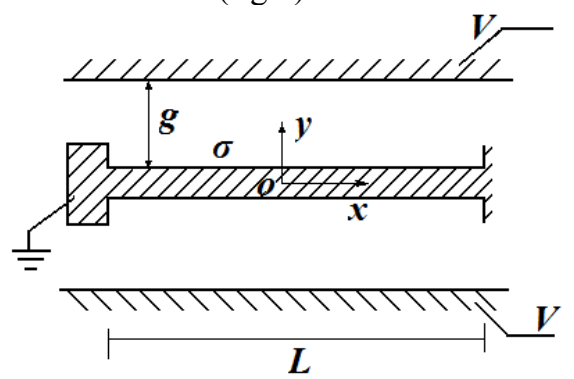

Fig.1. Clamped-clamped beam subject to an axially residual stress and a symmetric electrostatic field.

The effect of residual stress gradients is neglected but the residual stress is considered.

The electromechanical system is governed by the following nonlinear integral-differential equation [7], [9]:

$$
\begin{aligned}
& E^{*} I \frac{d^{4} y}{d x^{4}}-\sigma A \frac{d^{2} y}{d x^{2}}-E A\left[\frac{1}{L} \int_{-0.5 L}^{0.5 L}\left(\frac{d y}{d x}\right)^{2} d x\right] \frac{d^{2} y}{d x^{2}}= \\
& =\frac{1}{2} \varepsilon_{0} \lambda_{0} V^{2}\left[\frac{1}{(g-y)^{2}}-\frac{1}{(g+y)^{2}}\right]
\end{aligned}
$$

with the boundary conditions

$$
y(-0.5 L)=y(0.5 L)=\frac{d y}{d x}(-0.5 L)=\frac{d y}{d x}(0.5 L)=0
$$

Here $E^{*}=\frac{E}{\left(1-v^{2}\right)}$ is the effective modulus in bending,

$\mathrm{E}$ is the Young's modulus and $v$ is the Poisson ratio, $\mathrm{I}$ is the second moment of the beam cross-section, $b_{0}$ is the beam width and $\mathrm{h}$ is the beam thickness, $\mathrm{L}$ is the length, $\sigma$ is the axial residual stress, $\varepsilon_{0}$ is the permittivity of the free space, $A=b_{0} h$ is the cross-sectional area, $g$ is the nominal gap and $\mathrm{V}$ is the potential of the two fixed electrodes. It is assumed that $b>>h$ and $x \in[-\mathrm{L} / 2, \mathrm{~L} / 2]$ is the horizontal coordinate, while $\mathrm{y}$ is the transverse coordinate and denotes the post-buckling deflection of the beam. The three terms on the left hand side of Eq.(1) are the distributed mechanical forces associated with bending, axial stress and membrane stretching of the beam. The right side of Eq.(1) is the distributed electrostatic force.

Only symmetric modes of the deformed beam are considered and any possibility of symmetry and microbeam is disregarded.

The normalized form of the equilibrium equation can be expressed as

$$
\begin{aligned}
& Y^{(I V)}(s)-\tilde{\sigma} Y^{\prime \prime}(s)-12 \widetilde{E} r^{2}\left[\int_{0}^{0.5}\left(Y^{\prime}(s)\right)^{2} d s\right] Y^{\prime \prime}(s)= \\
& =U^{2} Y(s)\left[1-Y^{2}(s)\right]^{2}
\end{aligned}
$$

where prime denotes the derivative with respect to $\mathrm{s}$ and

$$
\begin{gathered}
s=\frac{x}{L}, \quad Y=\frac{y}{g}, \quad r=\frac{g}{h}, \quad \frac{\widetilde{\sigma}}{(2 \pi)^{2}}=\frac{\sigma}{\left|\sigma_{E}\right|}, \\
\widetilde{E}=\frac{E}{E^{*}}, \quad U^{2}=\frac{24 \varepsilon_{0} \lambda_{0} L^{4} V^{2}}{E^{*} g^{3} h^{3}}
\end{gathered}
$$

The Euler buckling stress is [7], [9]

$$
\sigma_{E}=-4 \pi^{2} E^{*} I /\left(A L^{2}\right)
$$

The boundary conditions of the beam are

$$
Y(-0.5)=Y(0.5)=Y^{\prime}(-0.5)=Y^{\prime}(0.5)=0
$$

Now, by introducing a new independent variable

$$
\tau=\frac{2 \pi x}{L}+\pi
$$

we transform Eqs. (3) and (6) into the following dimensionless form:

$$
\begin{gathered}
\left(1-Y^{2}\right)^{2}\left[Y^{(I V)}-\lambda Y^{\prime \prime}-\beta Y^{\prime \prime} \int_{0}^{2 \pi} Y^{\prime 2}(\tau) d \tau\right]-\mu Y=0 \\
Y(0)=Y(2 \pi)=Y^{\prime}(0)=Y^{\prime}(2 \pi)=0
\end{gathered}
$$


where

$$
\lambda=\frac{\widetilde{\sigma}}{(2 \pi)^{2}}, \quad \beta=\frac{3 \widetilde{E} r^{2}}{\pi}, \quad \mu=\frac{u^{2}}{2 \pi^{4}}
$$

\section{Basic ideas of the optimal homotopy asymptotic method (OHAM)}

The OHAM was proposed by Vasile Marinca and Nicolae Herisanu [19-24].

To apply OHAM, we consider the nonlinear differential equation in the general form

$$
L[Y(\tau)]+N[Y(\tau)]=0, \quad \tau \in D
$$

subject to the boundary/initial conditions

$$
B\left(Y(\tau), \frac{d Y}{d \tau}\right)=0
$$

where $\mathrm{L}$ is a linear operator, $\tau$ denotes independent variable, $\mathrm{Y}(\tau)$ is an unknown function, $\mathrm{N}[\mathrm{Y}(\tau)]$ is a nonlinear operator, $\mathrm{D}$ is the domain of interest and $\mathrm{B}$ is a boundary operator.

By means of OHAM, one constructs a family of equations

$$
\begin{aligned}
& (1-p) L\left(\tilde{Y}\left(\tau, C_{i}\right)\right]= \\
& =p H\left(\tau, C_{i}\right)\left[L \left(\widetilde{Y}\left(\tau, C_{i}\right)+N\left(\tilde{Y}\left(\tau, p, C_{i}\right)\right.\right.\right.
\end{aligned}
$$

where $\mathrm{p} \in[0,1]$ is an embedding parameter, $\mathrm{H}\left(\tau, \mathrm{C}_{\mathrm{i}}\right)$ is a nonzero auxiliary function for $\mathrm{p} \neq 0, \tilde{Y}\left(\tau, \mathrm{C}_{\mathrm{i}}\right)$ is an unknown function, $\mathrm{C}_{\mathrm{i}}, \mathrm{i}=1,2, \ldots, \mathrm{s}$ are $\mathrm{s}$ unknown parameters which will be optimally determined later. For $\mathrm{p}=0$ and $\mathrm{p}=1$ it holds

$$
\begin{aligned}
& \tilde{Y}\left(\tau, 0, C_{i}\right)=Y_{0}(\tau) \\
& \tilde{Y}\left(\tau, 1, C_{i}\right)=Y(\tau)
\end{aligned}
$$

Therefore, as $\mathrm{p}$ increases from 0 to 1 , the solution $\widetilde{Y}\left(\tau, p, C_{i}\right)$ varies from $\mathrm{Y}_{0}(\tau)$ to $\mathrm{Y}(\tau)$, where the initial approximation $\mathrm{Y}_{0}(\tau)$ is obtained from Eq.(1) for $\mathrm{p}=0$ :

$$
L\left[Y_{0}(\tau)\right]=0
$$

and

$$
B\left(Y_{0}, \frac{d Y_{0}}{d \tau}\right)=0
$$

Let us consider the solution of Eq.(13) in the form

$$
\widetilde{Y}\left(\tau, p, C_{i}\right)=Y_{0}\left(\tau+p Y_{1}\left(\tau, C_{i}\right)\right), \quad i=1,2, \ldots, s
$$

Substituting Eq. (18) into Eq. (15) and equating the coefficients of $\mathrm{p}^{\mathrm{o}}$ and $\mathrm{p}^{1}$, we obtain the governing equation of the initial approximation $\mathrm{Y}_{0}(\tau)$ given by Eq.(16) with the boundary/initial conditions (17) and then the governing equation of the first (and last) approximation $\mathrm{Y}_{1}$, i.e.

$$
L\left[Y_{1}\left(\tau, C_{i}\right)\right]=H\left(\tau, C_{i}\right) N_{0}\left[Y_{0}(\tau)\right]
$$

with

$$
B\left(Y_{1}, \frac{d Y_{1}}{d \tau}\right)=0
$$

where $\mathrm{N}_{\mathrm{m}}\left(\mathrm{Y}_{0}(\tau)\right)$ is the coefficient of $\mathrm{p}^{\mathrm{m}}$ obtained expanding $N\left[\widetilde{Y}\left(\tau, p, C_{i}\right)\right]$ in series with respect to the embedding parameter $\mathrm{p}$ :

$$
\begin{aligned}
& N\left[\tilde{Y}\left(\tau, p, C_{i)}\right]=N_{0}\left[Y_{0}(\tau)\right]+p N_{1}\left(Y_{0}(\tau), Y_{1}\left(\tau, C_{i}\right)+\ldots\right.\right. \\
& \left.+p^{m} N_{m}\left[Y_{0}(\tau), Y_{1}\left(\tau, C_{i}\right), \ldots, Y_{m}\left(\tau, C_{i}\right)\right]+\ldots\right)
\end{aligned}
$$

In our case, we consider only a single iteration and therefore we need to choose $\mathrm{m}=0$.

It should be emphasized that $Y_{0}$ and $Y_{1}$ are governed by the linear Eqs. (16) and (19) with the boundary/initial conditions given by Eqs.(17) and (20), respectively. The convergence of the solution (18) depends upon the auxiliary function $\mathrm{H}\left(\tau, \mathrm{C}_{\mathrm{i}}\right)$. There are some possibilities to choose the function $\mathrm{H}$ and it is important to mention that $\mathrm{H}\left(\tau, \mathrm{C}_{\mathrm{i}}\right)$ must follow the terms appearing in Eq.(19). Therefore, we try to choose $\mathrm{H}\left(\tau, \mathrm{C}_{\mathrm{i}}\right)$ so that in Eq. (19) the product $\mathrm{H}\left(\tau, \mathrm{C}_{\mathrm{i}}\right) \mathrm{N}_{0}\left[\mathrm{Y}_{0}(\tau)\right]$ be of the same shape with the terms which appear into $\mathrm{N}_{0}\left[\mathrm{Y}_{0}(\tau)\right]$.

If the sum (18) is convergent at $p=1$, one has

$$
\tilde{Y}\left(\tau, C_{i}\right)=Y_{0}(\tau)+Y_{1}\left(\tau, C_{i}\right)
$$

The Eq.(22) is the approximate solution of the first order.

Inserting Eq. (22) into Eq.(11), we obtain the residual

$$
\begin{gathered}
R\left(\tau, C_{i}\right)=L\left[\tilde{Y}\left(\tau, C_{i}\right)\right]+N\left[\widetilde{Y}\left(\tau, C_{i}\right)\right], \\
i=1,2, \ldots, s, \quad \tau \in D
\end{gathered}
$$

If $\mathrm{R}\left(\tau, \mathrm{C}_{\mathrm{i}}\right)=0$, then $\tilde{Y}\left(\tau, C_{i}\right)$ will be the exact solution, but this rarely happens to nonlinear problems. The convergence-control parameters $\mathrm{C}_{\mathrm{i}}, \mathrm{i}=1,2, \ldots \mathrm{s}$, may be optimally identified via various methods, like minimizing the square residual error, using:

$$
J\left(C_{1}, C_{2}, \ldots, C_{s}\right)=\int_{D} R^{2}\left(\tau, C_{1}, C_{2}, \ldots C_{s}\right) d \tau
$$

The values of the unknown parameters will be determined from the conditions:

$$
\frac{\partial J}{\partial C_{1}}=\frac{\partial J}{\partial C_{2}}=\ldots=\frac{\partial J}{\partial C_{s}}=0
$$

Alternatively, the system of algebraic equations necessary to identify the unknown convergence-control parameters may be obtained imposing the conditions

$$
R\left(\tau_{1}, C_{i}\right)=R\left(\tau_{2}, C_{i}\right)=\ldots=R\left(\tau_{s}, C_{i}\right)=0
$$

or by using the Galerkin method, the collocation method, the Ritz method, the Kantorovich method and so on. For 
more details see [20], [21].

With these optimal values of the convergence-control parameters known, the first-order approximate solution (22) is well determined.

\section{Application of OHAM to post-buckling deformation of MEMS}

We use the basic ideas of the OHAM by considering Eqs. (8) and (9). We may choose the linear operator in the form

$$
L[Y(\tau)]=Y^{I V}(\tau)+Y^{\prime \prime \prime}(\tau)
$$

We mention that, generally, the choosing of the linear operator is not unique. Taking into account (26), the Eqs. (16) and (17) become, respectively:

$$
\begin{gathered}
Y_{0}^{I V}(\tau)+Y_{0}^{\prime \prime}(\tau)=0 \\
Y_{0}(0)=Y_{0}(2 \pi)=Y_{0}^{\prime}(0)=Y_{0}^{\prime}(2 \pi)=0
\end{gathered}
$$

which have the following solution:

$$
Y_{0}\left(\tau, C_{1}\right)=C_{1}(1-\cos \tau)
$$

with $\mathrm{C}_{1}$ unknown for the moment.

The nonlinear operator $\mathrm{N}[\mathrm{Y}(\tau)]$ is obtained from Eqs. (8), (11) and (26):

$$
\begin{aligned}
& N[Y(\tau)]=-Y^{\prime \prime}+Y^{I V}\left(Y^{4}-2 Y^{2}\right)- \\
& -\left(1-Y^{2}\right)^{2}\left[\lambda+\beta\left(\int_{0}^{2 \pi} Y^{\prime 2} d \tau\right) Y^{\prime \prime}-\mu Y\right.
\end{aligned}
$$

such that substituting Eq. (29) into Eq. (30), we obtain

$$
\begin{aligned}
& N\left[Y\left(\tau, C_{i}\right)\right]=-C_{1}\left(1+\lambda+\pi \beta C_{1}^{2}\right)\left[M_{0}+M_{1} \cos \tau+\right. \\
& \left.+M_{2} \cos 2 \tau+M_{3} \cos 3 \tau+M_{4} \cos 4 \tau+M_{5} \cos 5 \tau\right]
\end{aligned}
$$

where

$$
\begin{aligned}
& M_{0}=\frac{\mu}{1+\lambda+\pi \beta C_{1}^{2}}+2 C_{1}^{2}-\frac{7}{2} C_{1}^{4}, \\
& M_{1}=-\frac{\mu}{1+\lambda+\pi \beta C_{1}^{2}}+1-\frac{7}{2} C_{1}^{2}+\frac{49}{8} C_{1}^{4} \\
& M_{2}=2 C_{1}^{2}-4 C_{1}^{4}, \quad M_{3}=\frac{29}{16} C_{1}^{4}-\frac{C_{1}^{2}}{2}, \\
& M_{4}=-\frac{C_{1}^{4}}{2}, \quad M_{5}=\frac{C_{1}^{4}}{16}
\end{aligned}
$$

Having in view that within Eq. (31) we can found a trigonometric function and that the auxiliary function $\mathrm{H}\left(\tau, \mathrm{C}_{\mathrm{i}}\right)$ must follow the terms appearing in Eq. (31), then we may choose the function $\mathrm{H}$ in the following forms:

$$
H\left(\tau, C_{i}\right)=-\frac{\left(C_{2}+2 C_{3} \cos \tau+2 C_{4} \cos 2 \tau\right)}{C_{1}\left(1+\lambda+\pi \beta C_{1}^{2}\right)}
$$

or

$$
H\left(\tau, C_{i}\right)=-\frac{1}{C_{1}\left(1+\lambda+\pi \beta C_{1}^{2}\right)}\left(C_{2}^{\prime}+2 C_{3}^{\prime} \cos \tau\right)
$$

or

$$
H=-\frac{C_{2}^{*}+2 C_{3}^{*} \cos \tau+2 C_{4}^{*} \cos 2 \tau+2 C_{5}^{*} \cos 3 \tau}{C_{1}\left(1+\lambda+\pi \beta C_{1}^{2}\right)}
$$

or yet

$$
H\left(\tau, C_{i}\right)=-\frac{\left(C_{2}^{\prime \prime}+2 C_{3}^{\prime \prime} \cos \tau+2 C_{4}^{\prime \prime} \cos 4 \tau\right)}{C_{1}\left(1+\lambda+\pi \beta C_{1}^{2}\right)}
$$

and so on, where $C_{i}, C_{i}^{\prime}, C_{i}^{\prime \prime}$ and $C_{i}^{*}$ are unknown parameters at this moment.

If we choose only the expression (33) for $\mathrm{H}\left(\tau, \mathrm{C}_{\mathrm{i}}\right)$, then using (31), (33) and (19) we can obtain the equation in the first approximation $Y_{1}$ :

$$
\begin{aligned}
& Y_{1}^{I V}+Y_{1}^{\prime \prime}=M_{0} C_{2}+M_{1} C_{3}+M_{2} C_{4}+\left[M_{1}\left(C_{2}+C_{4}\right)\right. \\
& \left.+2 M_{0} C_{3}+M_{2} C_{3}+M_{3} C_{4}\right] \cos \tau+\left[M_{2} C_{2}+\left(M_{1}+\right.\right. \\
& \left.\left.+M_{3}\right) C_{3}+\left(2 M_{0}+M_{4}\right) C_{4}\right] \cos 2 \tau+\left[M_{3} C_{2}+\left(M_{2}+\right.\right. \\
& \left.\left.+M_{4}\right) C_{3}+\left(M_{1}+M_{5}\right) C_{4}\right] \cos 3 \tau+\left[M_{4} C_{2}+\left(M_{3}+\right.\right. \\
& \left.\left.+M_{5}\right) C_{3}+M_{2} C_{4}\right] \cos 4 \tau+\left(M_{5} C_{2}+M_{4} C_{3}+\right. \\
& \left.+M_{3} C_{4}\right) \cos 5 \tau+\left(M_{5} C_{3}+M_{4} C_{4}\right) \cos 6 \tau+ \\
& +M_{5} C_{4} \cos 7 \tau \\
& Y_{1}(0)=Y_{1}(2 \pi)=Y_{1}^{\prime}(0)=Y_{1}^{\prime}(2 \pi)=0
\end{aligned}
$$

No secular terms in $\mathrm{Y}_{1}$ requires

$$
M_{1}\left(C_{2}+C_{4}\right)+2 M_{0} C_{3}+M_{2} C_{3}+M_{3} C_{4}=0
$$

The solution of Eq. (37) can be written as

$$
\begin{aligned}
& Y\left(\tau, C_{1}, C_{2}, C_{3}, C_{4}\right)= \\
& \frac{M_{2} C_{2}+\left(M_{1}+M_{3}\right) C_{3}+\left(2 M_{0}+M_{4}\right) C_{4}}{12}(\cos 2 \tau-\cos \tau)+ \\
& +\frac{M_{3} C_{2}+\left(M_{2}+M_{4}\right) C_{3}+\left(M_{1}+M_{5}\right) C_{4}}{72}(\cos 3 \tau-\cos \tau)+ \\
& +\frac{M_{4} C_{2}+\left(M_{3}+M_{5}\right) C_{3}+M_{2} C_{4}}{240}(\cos 4 \tau-\cos \tau)+ \\
& +\frac{M_{5} C_{2}+M_{3} C_{4}}{600}(\cos 5 \tau-\cos \tau)+\frac{M_{5} C_{3}+M_{4} C_{4}}{1260}(\cos 6 \tau- \\
& -\cos \tau)+\frac{M_{5} C_{4}}{2352}(\cos 7 \tau-\cos \tau)
\end{aligned}
$$

The first-order approximate solution of Eqs. (8) and (9) is obtained from Eqs. (29), (39) and (22):

$$
\widetilde{Y}\left(\tau, C_{1}, C_{2}, C_{3}, C_{4}\right)=Y_{0}\left(\tau, C_{1}\right)+Y_{1}\left(\tau, C_{1}, C_{2}, C_{3}, C_{4}\right)
$$

\section{Numerical example}

In order to show the validity of the proposed procedure, we consider the following values of the parameters which are involved in Eq.(8): 


$$
\lambda=-0.96, \quad \beta=\frac{4.32}{\pi}, \quad \mu=0.025
$$

Applying the described procedure for minimizing the residual, the optimal values of the convergence-control parameters are obtained as:

$$
\begin{aligned}
& C_{1}=0.083871487738863, \mathrm{C}_{2}=0.00100704014882 \\
& \mathrm{C}_{3}=-0.00062148786228, C_{4}=0.00100759265364
\end{aligned}
$$

so that the approximate solution of Eqs.(8) and (9) can be obtained in the form:

$$
\begin{aligned}
& Y(\tau)=0.083871487738-0.083911246033 \cos \tau+ \\
& +0.000031180822 \cos 2 \tau+8.516244 \cdot 10^{-6} \cos 3 \tau+ \\
& +6.6998 \cdot 10^{-8} \cos 4 \tau-5.7507 \cdot 10^{-9} \cos 5 \tau- \\
& -2.131 \cdot 10^{-11} \cos 6 \tau+1.32 \cdot 10^{-12} \cos 7 \tau
\end{aligned}
$$

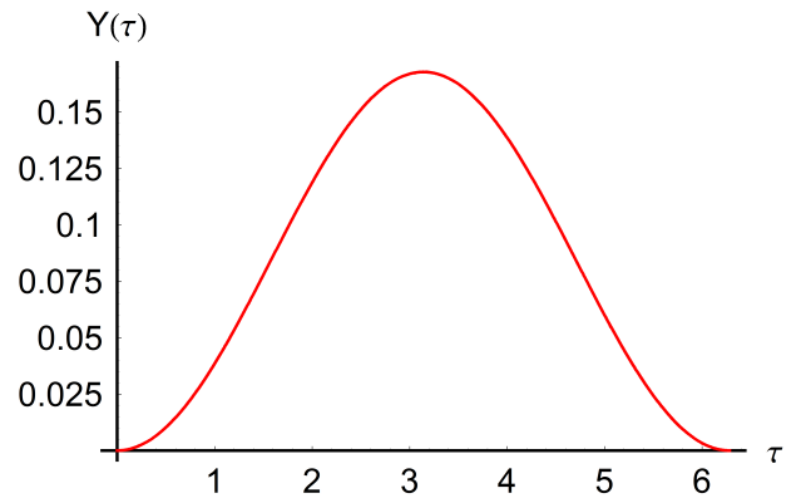

Fig.2. Approximate solution (43) of Eqs. (8) and (9)

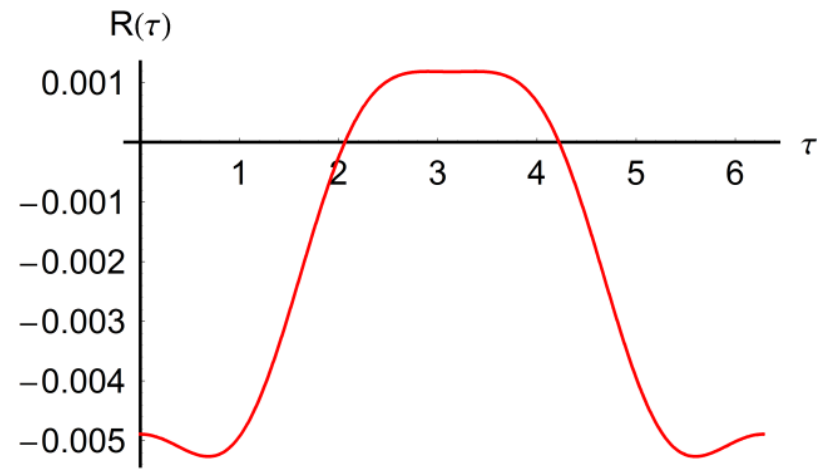

Fig.3. Residual (23) of the approximate solution (43)

In fig. 2 we present the approximate solution (43) of Eqs. (8) and (9) obtained through OHAM while in fig.3 it is presented the residual (13) obtained for the approximate solution (43). It can be observed the accuracy of the solution, since the obtained residual is less than 0.005 .

\section{Conclusions}

In this study we proposed an analytical approximate solution to the electromechanical post-buckling response of an axially stressed clamped-clamped actuator, modeled as a beam, subjected to a symmetric electrostatic field.
Our procedure is valid even if the nonlinear differential equation does not contain any small or large parameters. In the construction of the proposed homotopy appear some distinctive concepts such as the auxiliary function $\mathrm{H}\left(\tau, \mathrm{C}_{\mathrm{i}}\right)$ and several convergencecontrol parameters $C_{i}$ whose optimal values ensure a fast convergence of the solution.

The example presented in this work lead to the conclusion that the obtained results are quite accurate after the first iteration. This technique is effective, explicit and accurate for a nonlinear integral-differential equation. Our construction of homotopy is different from other approaches, especially in what concerns the linear operator $\mathrm{L}$ and the auxiliary convergence-control function $\mathrm{H}$.

As it was demonstrated, the proposed method is straightforward, concise, and can be applied to other nonlinear problems.

\section{References}

1. S.B. Yamgoue, A.J. Tchiegang, J. Nonlinear Dynamics, ID 735712 (2014)

2. H.C. Nathanson, W.E, Newell, R.A. Wickstrom, T.R. Davis Jr., IEEE Transactions on Electron Devices, 14, 117 (1996)

3. M. Elwespoek, R. Wiegerink, Mechanical microsensors (Springer, Berlin, 2001)

4. V.M Varadan, K.J. Visroy, K.A. Jose, RF MEMES and their applications (Wiley, NY, 2003)

5. M.I. Younis, E.M. Abdel Rehman, A. Nayfeh, J. Microelectromechanical Syst., 12, 672 (2003)

6. A.H. Nayfeh, M.I. Younis, E.M. Abdel Rehman, Nonlinear Dynamics, 48, 153 (2007)

7. S. Abu-Salih, D. Elata, Using electromechanical buckling for measuring residual stress, EuroSime 2006 - 7th International Conference on Thermal, Mechanical and Multiphysics Simulation and Experiments in Micro-Electronics and MicroSystems, DOI: 10.1109/ESIME.2006.1643991

8. Y-C Hu, J. Micromech. Microeng., 16, 648 (2006)

9. Y Yu, B.S. Wu, C.W. Lim, Int. J Mech. Sci., 55, 95 (2012)

10. G. Rezazadeh, M. Fathalilou, M. Sadeghi, Imaging, 12, 117 (2011)

11. S. Chaterjee, G. Pohit, Int. J. Eng. Sci. Techn., 2, 187 (2010)

12. Y. Zhu, H.D. Espinoza, J. Micromech. Microeng., 14, 1270 (2004)

13. Y.T. Beni, M. Heidari, Int. J. Eng. Appl. Sci., 4, 41 (2012)

14. M. Mojahedi, M. Moghimi Zand, M.T. Ahmadian, Appl. Math. Modelling, 34, 1032 (2010)

15. S.S. Singh, P. Pal, A.K. Pandey, J. of Vibration and Acoustics, 138, 064502 (2016)

16. D.I Caruntu, I. Martinez, K.N. Taylor, Mech, Research Commun., 52, 25 (2013) 
17. H. Daneshpajooh, M. Moghimi Zand, J. of Mech. Sci. and Technology, 29, 3855 (2015)

18. C.S. Harsha, C.S.R. Prasanth, B. Pratiher, Procedia Engineering, 14, 891 (2016)

19. N. Herisanu, V. Marinca, T. Dordea, Gh. Madescu, Proc. Rom. Acad, Ser.A, 9, 229 (2008)

20. V. Marinca, N. Herisanu, J. Sound Vibr. 329, 1450 (2010)

21. V. Marinca, N. Herisanu, Nonlinear dynamical systems in engineering. Some approximate approaches (Springer, Heidelberg, 2011)

22. V. Marinca, N. Herisanu, The optimal homotopy asymptotic method. Engineering applications (Springer, Cham, 2015)

23. N. Herisanu, V. Marinca, Gh. Madescu, Wind Energy, 18, 1657 (2015)

24. V. Marinca, N. Herisanu, Int. J. Heat and Mass Transf 79, 146 (2014) 